\title{
Clinicopathological Features of Metastatic Gastric Tumors Originating From Breast Cancer: Analysis of Eleven Cases
}

\author{
Yuta Ushida ${ }^{\mathrm{a}}$, Shoichi Yoshimizu ${ }^{\mathrm{b}, \mathrm{c}}$, Yusuke Horiuchi ${ }^{\mathrm{b}}$, Toshiyuki Yoshio ${ }^{\mathrm{b}}$, \\ Akiyoshi Ishiyama ${ }^{\mathrm{b}}$, Toshiaki Hirasawa ${ }^{\mathrm{b}}$, Tomohiro Tsuchida ${ }^{\mathrm{b}}$, \\ Junko Fujisaki ${ }^{b}$
}

\begin{abstract}
Background: Metastatic gastric tumor originating from breast cancer (MGTBC) is rare. Endoscopically, gastric cancer (GC)-like lesions and submucosal tumors (SMT) are the features of MGTBC. Their clinicopathological characteristics are currently uncertain. We analyzed the clinicopathological characteristics of 11 patients with MGTBC to better understand the disease progression and thereby improve early detection methods.
\end{abstract}

Methods: From 2006 to 2016, 11 patients with MGTBC diagnosed by esophagogastroduodenoscopy (EGD) were included.

Results: All 11 patients were women, with a median age of 57 years. Histological examination revealed six cases with solid tubular components and five cases with invasive lobular carcinoma components. Melena was the most common symptom; however, asymptomatic cases were also common. Seven and four cases had GC-like lesions and SMTs, respectively. Six of the seven cases had GC-like lesions with peritoneal metastasis, whereas none of the four SMT cases had peritoneal metastasis $(\mathrm{P}=0.015)$. The median overall survival (OS) in all cases was 26 months (range, 1 - 42 months). OS in cases treated with chemotherapy after a diagnosis of gastric metastases was significantly better than that in those treated without chemotherapy $(\mathrm{P}$ $=0.047$ ). One patient showed gradual transformation from an SMTtype lesion to a Borrmann type 4-like appearance.

Conclusions: MGTBC is a rare occurrence; however, it should be considered when gastric tumors, especially GC-like lesions in patients with a medical history of breast cancer are found. Patients with breast cancer, especially invasive lobular carcinoma, should undergo

Manuscript submitted May 22, 2018, accepted August 6, 2018

aDepartment of Gastroenterological Surgery, Cancer Institute Hospital of the Japanese Foundation for Cancer Research, 3-8-31 Ariake, Koto-ku, Tokyo 135-8550, Japan

bDepartment of Gastroenterology, Cancer Institute Hospital of the Japanese Foundation for Cancer Research, 3-8-31 Ariake, Koto-ku, Tokyo 135-8550, Japan

${ }^{\mathrm{c}}$ Corresponding Author: Shoichi Yoshimizu, Department of Gastroenterology, Cancer Institute Hospital of the Japanese Foundation for Cancer Research, 3-8-31 Ariake, Koto-ku, Tokyo 135-8550, Japan.

Email: shoichi.yoshimizu@jfcr.or.jp

doi: https://doi.org/10.14740/wjon1112w screening EGD regularly to detect gastric metastases early and receive chemotherapy to obtain good outcomes.

Keywords: Gastric tumor; Metastatic gastric tumor; Breast cancer; Submucosal tumor; Borrmann type 4 lesion; Endoscopy

\section{Introduction}

Metastatic gastric tumor originating from breast cancer (MGTBC) is a rare clinical entity [1] and its clinicopathological features have not yet been clarified. Several studies have reported that the most common histological type of MGTBC is invasive lobular carcinoma (ILC), although it is less common than infiltrating ductal carcinoma (IDC) in primary breast cancer $[1,2]$. The endoscopic appearance of MGTBC is classified into two types, gastric cancer (GC)-like lesions and submucosal tumors (SMT) [3]. There have been no reports of relationships between endoscopic characteristics and clinical features in either of the types so far. Diagnostically, it is sometimes difficult to determine whether a GC-like lesion is a gastric metastatic tumor or a primary gastric tumor, especially a Borrmann type 4 lesion. The treatment strategy is different for primary GC and MGTBC, so it is important to diagnose them precisely. Generally, MGTBC is treated with systemic therapy based on Stage IV breast cancer treatment [4]; however, there is no evidence-based therapy available for this treatment method. In this study, we observed the endoscopic appearances and clinical courses in 11 patients with MGTBC and analyzed their clinicopathological features.

\section{Materials and Methods}

From 2006 to 2016, we performed 57,952 cases of esophagogastroduodenoscopy (EGD), and there were 11 patients $(0.019 \%)$ with MGTBC who were treated at the Cancer Institute Hospital of the Japanese Foundation for Cancer Research. All the gastric lesions were diagnosed as MGTBC by histology. We investigated their clinicopathological characteristics by medical chart review (age, sex, complaint, TNM stage of primary breast cancer, histological type, endoscopic findings, metastatic site, hormonal receptor status of speci- 
Table 1. Characteristics of Primary Breast Cancer Patients

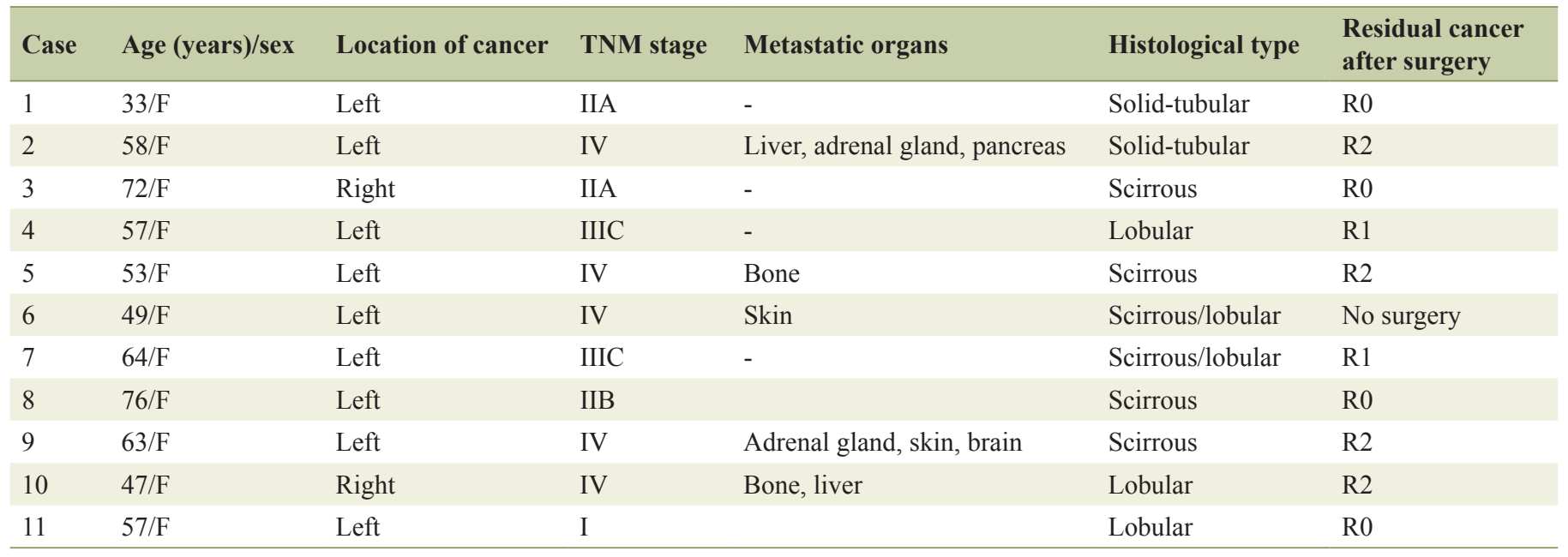

F: female.

men, and survival outcome). The presence of oligometastatic breast cancer was determined based on clinical evaluation and systemic radiographic imaging demonstrating fewer than five metastatic lesions. All patients provided comprehensive written informed consent. The institutional review board of the Cancer Institute Hospital of the Japanese Foundation for Cancer Research approved this study and it was conducted in accordance with the Declaration of Helsinki 1964 and its later amendments.

Statistical analysis was carried out using EZR (Saitama Medical Center, Jichi Medical University, Saitama Japan) which is a graphical user interface for R (The R Foundation for Statistical Computing, Vienna, Austria). More precisely, it is a modified version of $\mathrm{R}$ commander designed to add statistical functions frequently used in biostatistics. We compared the categorical parameters using the Fisher's exact test and continuous parameters using the Mann-Whitney U-test. Overall survival (OS) was calculated from the date of diagnosis of gastric metastasis until death from any cause or the last followup date. The time to survival curve was established using the Kaplan-Meier method. In all analyses, P-value less than 0.05 was determined to be statistically significant.

\section{Results}

\section{Characteristics of primary breast cancer}

All 11 patients were women, with a median age of 57 years (range, 33 - 76 years, Table 1). Histological examination revealed six cases $(54.5 \%)$ with solid tubular components and five cases $(45.5 \%)$ with ILC components. Five patients (45.5\%) had Stage IV disease according to the Union for International Cancer Control, 7th edition (UICC7th) and four patients $(36.4 \%)$ underwent curative resection. There were no significant differences between patients underwent curative mastectomy and patients with residual cancer after mastec- tomy in median survival time from diagnosis of primary breast cancer (median survival time; 163 vs. 89.8 months, Hazard ratio; $2.14, \mathrm{P}=0.399$ ) and from gastric metastasis (median survival time; 16.0 vs. 37.2 months, Hazard ratio; $0.187, \mathrm{P}=$ 0.146).

\section{Clinical characteristics and prognosis of MGTBC}

Among the clinical symptoms of MGTBC, melena was the most common (27.3\%); however, asymptomatic cases were also common (Table 2$)$. Three patients $(27.3 \%)$ had upper abdominal symptoms of epigastric pain and heartburn. Gastric specimens from nine patients were subjected to a hormonal receptor test, and all were positive for the estrogen receptor (ER). Seven patients of human epidermal growth factor receptor 2 (HER2) with immunohistochemistry test were all negative. Seven and four cases had GC-like lesions and SMTs, respectively (Fig. 1). Of the 11 cases, six had Borrmann type 4-like lesions. The median period from primary breast cancer diagnosis to the detection of gastric metastases was 60 months (range, 19 - 178 months). The median OS in all patients was 26 months (range, 1 - 42 months; Fig. 2), and that in the patients treated with chemotherapy after the diagnosis of gastric metastases was significantly better than that in those who did not receive chemotherapy with supportive care (median OS; 28.9 vs. 3.80 months, Hazard ratio; 8.27, $\mathrm{P}=0.047$ ). Oligometastatic breast cancer (metastatic lesions; $\mathrm{n} \leq 5$ ) also has significantly better outcome than those which had more than six metastatic lesions (median OS; 39.4 vs. 26.4 months, Hazard ratio; 3.17, $\mathrm{P}=0.0239)$.

\section{GC-like lesion vs. SMT}

We compared GC-like lesions with SMTs. We analyzed the effect of age, presence or absence of symptoms, histology, number of lesions, and whether or not chemotherapy was per- 


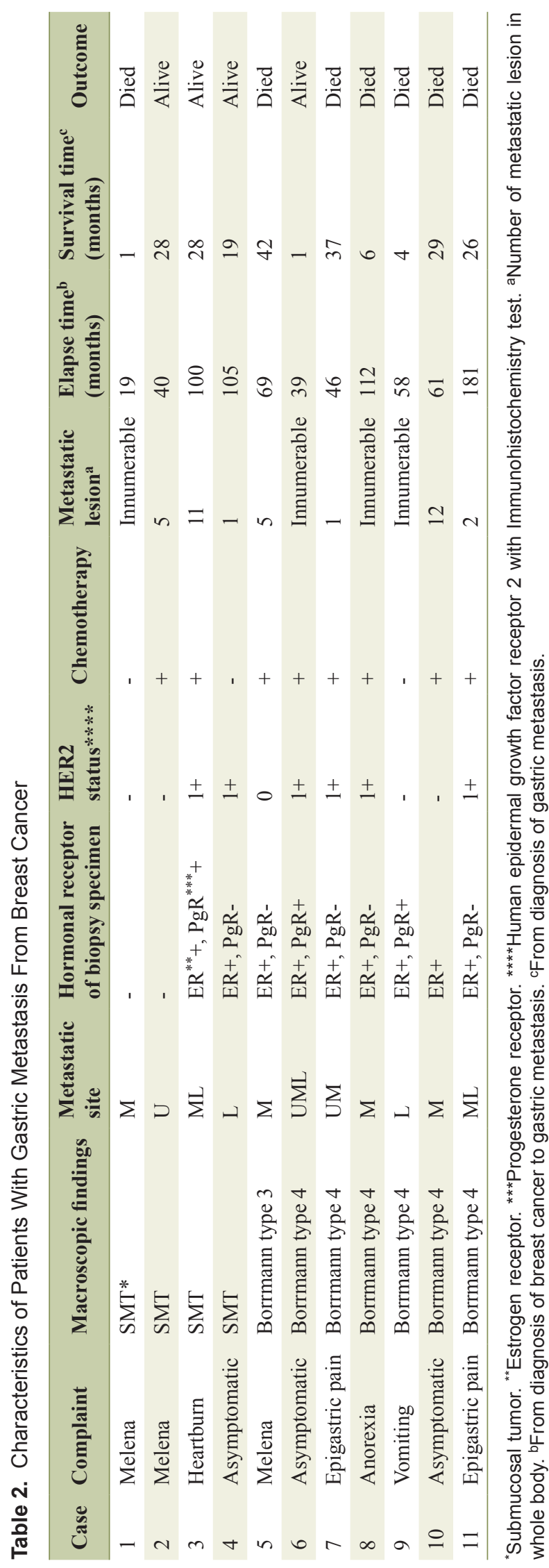

formed after a diagnosis of MGTBC; there were no significant differences in OS with any of the factors. Six of the seven cases had GC-like lesions with peritoneal metastasis, whereas none of the SMT cases had peritoneal metastasis $(\mathrm{P}=0.015$, Table 3).

\section{A case with transformation of the endoscopic appearance from SMT to a GC-like lesion}

On temporal observation, there was a patient whose lesion showed gradual transformation from an SMT-type lesion to a lesion with a Borrmann type 4-like appearance.

In case No. 4, a 57-year-old woman was found to have a gastric tumor using EGD during a regular health check-up. Her height was $155 \mathrm{~cm}$, weight was $56 \mathrm{~kg}$, and body surface area was $1.54 \mathrm{~m}^{2}$. Her medical history included breast cancer surgery (pT3pT3M0, pStage IIIC according to UICC 7th) 105 months ago and adjuvant chemotherapy with CAF (cyclophosphamide $\left(500 \mathrm{mg} / \mathrm{m}^{2}\right.$ intravenously (IV), day 1$)$, doxorubicin $\left(50 \mathrm{mg} / \mathrm{m}^{2} \mathrm{IV}\right.$, day 1$)$, and 5 -fluorouracil $\left(500 \mathrm{mg} / \mathrm{m}^{2} \mathrm{IV}\right.$, day 1)) in six courses without severe adverse effects. In our examination, EGD demonstrated an SMT lesion in the greater curvature of the gastric antrum (Fig. 3A, B). One year later, EGD showed enlargement of this lesion and thickening of the wall in lesser curvature of gastric angle (Fig. 3C, D). One and a half years later, EGD revealed stenosis of the lumen in the gastric antrum and diffuse thickening of the gastric wall that resembled Borrmann type 4 disease (Fig. 3E, F). Simultaneously, computed tomography (CT) showed the presence of a peritoneal metastasis. The patient has survived and is currently receiving palliative care.

\section{Discussion}

The incidence of MGTBC is estimated to be approximately $11.6 \%$ based on the results of autopsy series on patients with breast cancer. Nevertheless, gastric metastases are uncommon entities in clinical practice and were only identified in $3.5 \%$ of the aforementioned patients while they were alive [3]. Histologically, MGTBC are common in ILC. Metastasis to the lung, liver, bone, and brain from breast cancer are more common for IDC than for ILC. In addition, it is more common for ILC than IDC to metastasize to the gastrointestinal tract, peritoneum, and ovary [5]. In Japan, MGTBC is especially rare, as the incidence of ILC (3.7\%) is lower than that in the United States $(8-14 \%)[1,2,6]$.

As shown in Table 2, some patients with gastric metastases were asymptomatic, whereas others had nonspecific symptoms; therefore, it was difficult to diagnose gastric metastases only on the basis of clinical symptoms. The median OS of patients with MGTBC was 11 months in a previous study [7], and the clinical result in our hospital (median OS; 26 months) was reasonable. The prognosis of MGTBC is unfavorable because the gastrointestinal tract is not a frequent site of metastasis generally and, more often than not, gastric metastasis develops at an advanced stage of breast cancer. Oligometastatic disease 

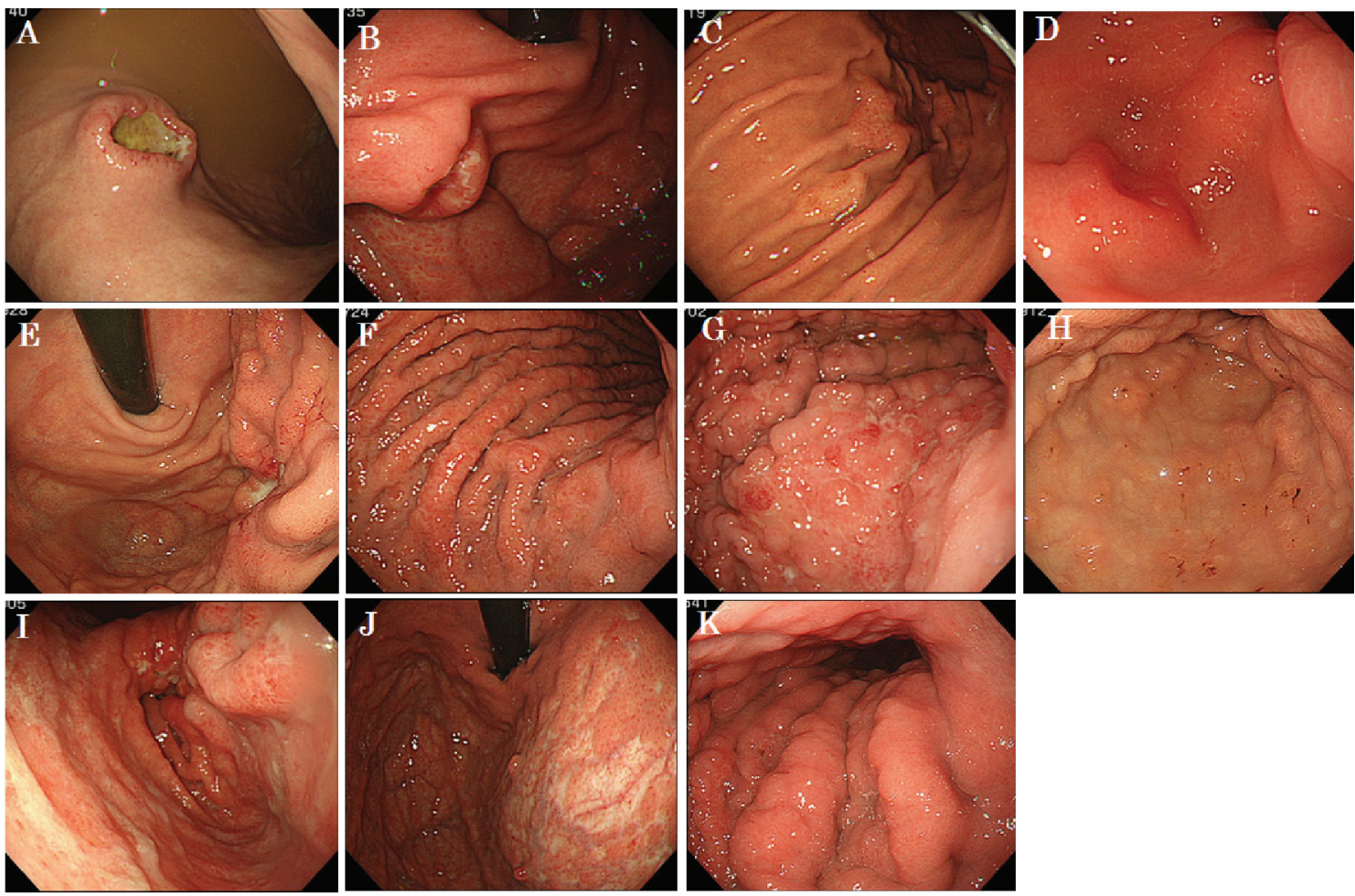

Figure 1. White light endoscopic features of 11 patients with gastric metastasis of breast cancer.

has better prognosis than widely metastatic disease [8]. The same can be said of MGTBC. The outcomes for the four patients that underwent potentially curative resection were not obvious, and curative mastectomy and mastectomy as local therapy had no significant difference in outcome. This suggests that local therapy to control the primary tumor might have equivalent benefit to curative mastectomy for oligomeric breast cancer. It is important to study some more cases with

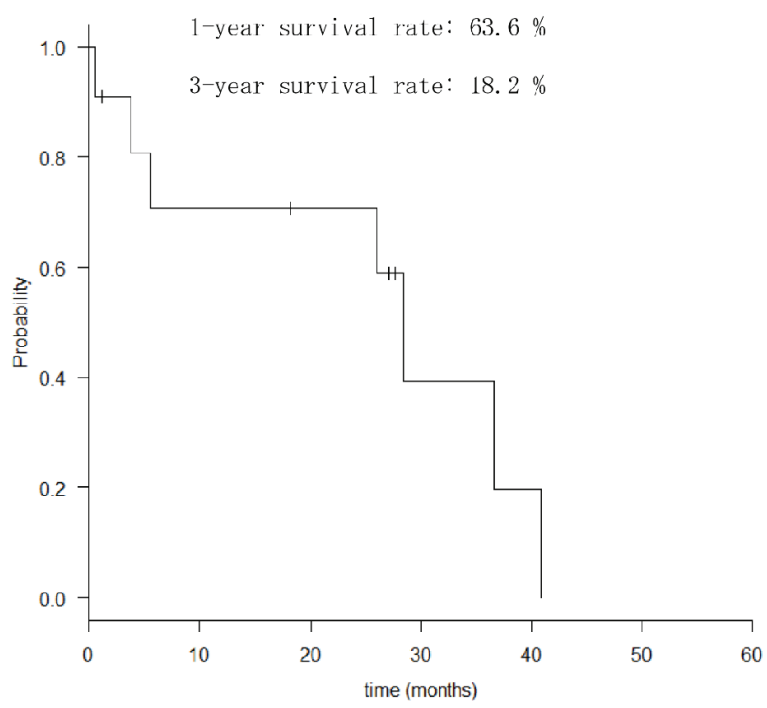

Figure 2. Overall survival rates of patients with gastric metastasis. similar outcomes to establish its characteristics. The median OS in the patients treated with chemotherapy was significantly better than that in those who did not receive chemotherapy; however, this result includes factors that may lead to bias, such as age, performance status, staging of breast cancer, and comorbidity. Early detection of MGTBC is important to allow a range of treatment options, and an early diagnosis means that chemotherapy and local therapy can be performed with good outcomes.

A well-known finding for gastric metastasis in radiography is the bull's eye sign; this corresponds to the endoscopic appearance of SMT, which has a central depression. However, in our study, seven of the 11 cases had lesions with a GC-like macroscopic appearance especially that of a Borrmann type 4-like lesion. Although, there are difficulties with the macroscopic differential diagnosis in Borrmann type 4 lesions, the differential diagnosis of MGTBC and GC is significant for treatment. In general, MGTBC is treated using systemic chemotherapy (antibiotics, plant alkaloids, and monoclonal antibodies) as Stage IV breast cancer based on age, performance status, ER and HER2 status [9-11]. In contrast, GC is treated with surgical therapy if it is resectable without distant metastasis. It is essential to confirm a past medical history of breast cancer at the time of diagnosis. However, in four cases, the time from primary breast cancer diagnosis to the detection of gastric metastases was more than 100 months, which was sometimes too long to perform effective treatment. Histologically, ILC has a signet ring cell morphology, which can be confused with a primary gastric adenocarcinoma with poor differentiation [12]. Owing to an 
Table 3. Comparison of Characteristics Between Gastric Cancer (GC)-Like Lesion and SMT

\begin{tabular}{|c|c|c|c|}
\hline & GC -like lesion $(\mathrm{N}=7)$ & SMT $(\mathbf{N}=4)$ & P value \\
\hline Age, years, median (range) & $57(49-76)$ & $57(33-72)$ & 0.925 \\
\hline Symptomatic (\%) & $5(71)$ & $3(75)$ & 1 \\
\hline Invasive lobular carcinoma (\%) & $4(57)$ & $1(25)$ & 0.545 \\
\hline Multiple lesions (\%) & $6(86)$ & $2(50)$ & 0.491 \\
\hline Elapse time from diagnosis of breast cancer to gastric metastases, months, median (range) & $61(39-181)$ & $70(19-106)$ & 0.571 \\
\hline Survival time from gastric metastasis, months, median (range) & $26(4-37)$ & $23(1-28)$ & 0.571 \\
\hline
\end{tabular}

accurate histological diagnosis, deep biopsies in endoscopy are needed to obtain an appropriate specimen to distinguish MGTBC from GC because the main lesion in MGTBC is located in the submucosa [3]. Moreover, immunohistochemical analysis can be helpful in reaching the final diagnosis. A metastatic tumor in any organ from breast cancer is generally positive for cytokeratin (CK) 7, gross cystic disease fluid protein (GCDFP)-15, ER and progesterone receptor $(\mathrm{PgR})$, and negative for CK20 [13, 14].

We experienced a case in which the endoscopic appearance of a lesion changed from SMT to Borrmann type 4 during follow-up. This suggests that MGTBC initially have a SMTtype finding. As the tumor advances, the tumor cells gradually develop in the submucosa, forming a GC-like lesion that is often accompanied by peritoneal metastasis. In this case, peritoneal dissemination was confirmed using CT when the morphologic change from SMT to Borrmann type 4 was endoscop- ically ascertained. Cases with Borrmann type 4 lesions also clearly have more peritoneal metastasis than those with SMT.

The present study has limitations as a result of the small number of cases. More cases need to be studied to establish the characteristics of MGTBC.

In conclusion, MGTBC is a rare entity; however, we should consider it when we find gastric tumors, especially Borrmann type 4 lesions in patients with a medical history of breast cancer. Patients with breast cancer, particularly ILC, should undergo screening EGD regularly to detect gastric metastases early and receive appropriate therapy to obtain good outcomes.

\section{Conflict of Interest}

The authors declare that they have no competing interest. No

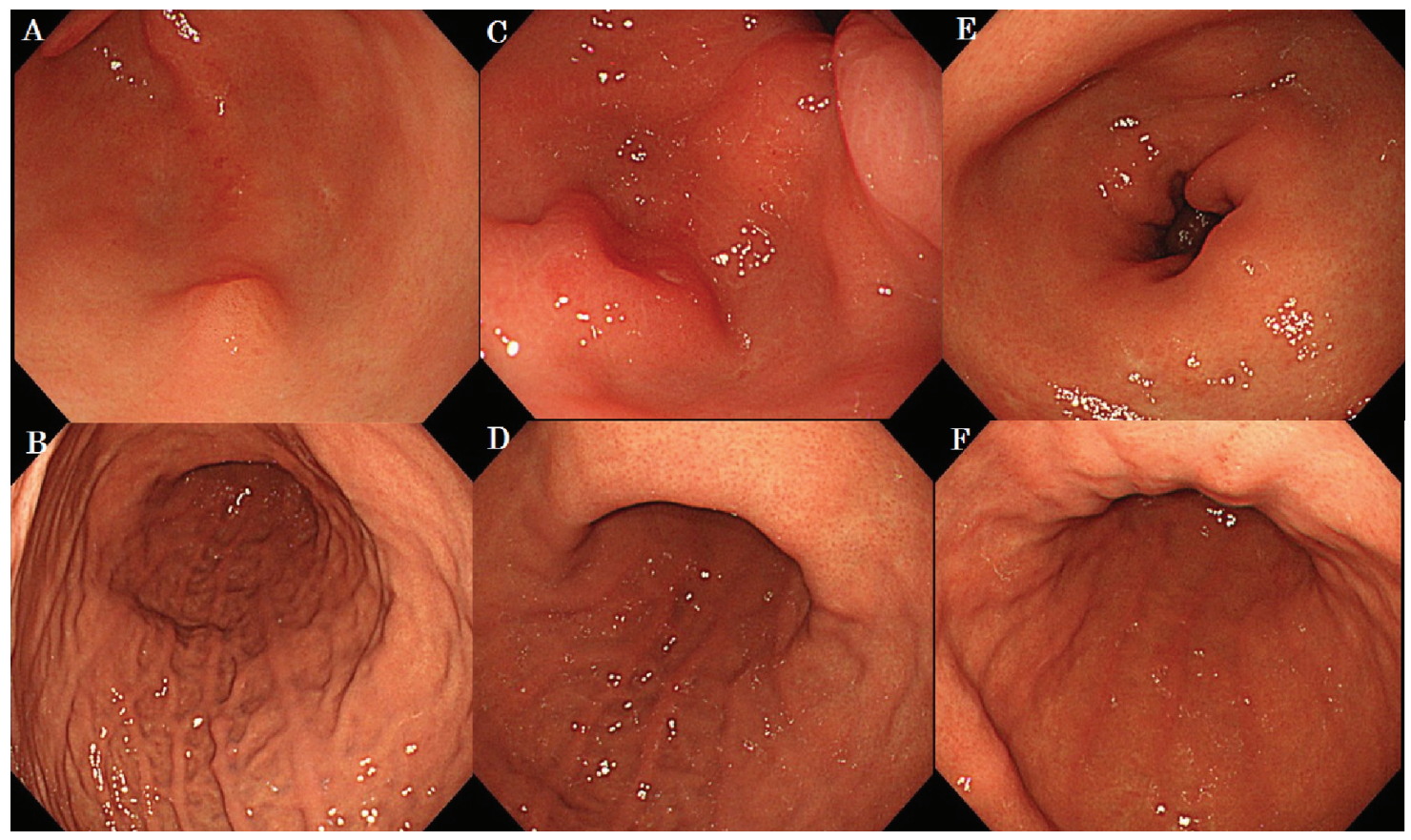

Figure 3. The case of macroscopic type progressed from submucosal tumor to Borrmann type 4. 
financial support has been received.

\section{Funding}

The authors declare that they have no funding.

\section{Author Contributions}

YU drafted the manuscript. YU and SY conceived the idea for the paper and helped draft the manuscript. JF proofread the paper. All the authors read and approved the final version of the manuscript.

\section{Abbreviations}

MGTBC: metastatic gastric tumor originating from breast cancer; ILC: invasive lobular carcinoma; IDC: infiltrating ductal carcinoma; GC: gastric cancer; SMT: submucosal tumor; EGD: esophagogastroduodenoscopy; OS: overall survival; CK: cytokeratin; GCDFP: gross cystic disease fluid protein

\section{References}

1. Borst MJ, Ingold JA. Metastatic patterns of invasive lobular versus invasive ductal carcinoma of the breast. Surgery. 1993;114(4):637-641; discussion 641-632.

2. Martinez V, Azzopardi JG. Invasive lobular carcinoma of the breast: incidence and variants. Histopathology. 1979;3(6):467-488.

3. Oda, Kondo H, Yamao T, Saito D, Ono H, Gotoda T, Yamaguchi H, et al. Metastatic tumors to the stomach: analysis of 54 patients diagnosed at endoscopy and 347 autopsy cases. Endoscopy. 2001;33(6):507-510.

4. Gennari A, Conte P, Rosso R, Orlandini C, Bruzzi P. Survival of metastatic breast carcinoma patients over a 20-year period: a retrospective analysis based on individual patient data from six consecutive studies. Cancer. 2005;104(8):1742-1750.

5. Arpino G, Bardou VJ, Clark GM, Elledge RM. Infiltrating lobular carcinoma of the breast: tumor characteristics and clinical outcome. Breast Cancer Res. 2004;6(3):R149156.

6. Japanese Breast Cancer Society. The report of clinical statistical studies on registrated mammary cancer patients in Japan.

7. Pectasides D, Psyrri A, Pliarchopoulou K, Floros T, Papaxoinis G, Skondra M, Papatsibas G, et al. Gastric metastases originating from breast cancer: report of 8 cases and review of the literature. Anticancer Res. 2009;29(11):4759-4763.

8. Leeman JE, Li JG, Pei X, Venigalla P, Zumsteg ZS, Katsoulakis E, Lupovitch E, et al. Patterns of treatment failure and postrecurrence outcomes among patients with locally advanced head and neck squamous cell carcinoma after chemoradiotherapy using modern radiation techniques. JAMA Oncol. 2017;3(11):1487-1494.

9. Norris B, Pritchard KI, James K, Myles J, Bennett K, Marlin S, Skillings J, et al. Phase III comparative study of vinorelbine combined with doxorubicin versus doxorubicin alone in disseminated metastatic/recurrent breast cancer: National Cancer Institute of Canada Clinical Trials Group Study MA8. J Clin Oncol. 2000;18(12):23852394.

10. O'Shaughnessy J, Miles D, Vukelja S, Moiseyenko V, Ayoub JP, Cervantes G, Fumoleau P, et al. Superior survival with capecitabine plus docetaxel combination therapy in anthracycline-pretreated patients with advanced breast cancer: phase III trial results. J Clin Oncol. 2002;20(12):2812-2823.

11. Dawood S, Broglio K, Buzdar AU, Hortobagyi GN, Giordano SH. Prognosis of women with metastatic breast cancer by HER2 status and trastuzumab treatment: an institutional-based review. J Clin Oncol. 2010;28(1):92-98.

12. Taal BG, Peterse H, Boot H. Clinical presentation, endoscopic features, and treatment of gastric metastases from breast carcinoma. Cancer. 2000;89(11):2214-2221.

13. Chu PG, Weiss LM. Immunohistochemical characterization of signet-ring cell carcinomas of the stomach, breast, and colon. Am J Clin Pathol. 2004;121(6):884-892.

14. Tot $\mathrm{T}$. The role of cytokeratins 20 and 7 and estrogen receptor analysis in separation of metastatic lobular carcinoma of the breast and metastatic signet ring cell carcinoma of the gastrointestinal tract. APMIS. 2000;108(6):467-472. 